\title{
NOVEL, CONTINUOUS VISUAL MOTION INDUCES $c$-fOS EXPRESSION IN THE AVIAN OPTOKINETIC NUCLEI AND OPTIC TECTUM
}

\author{
X. ROJAS, ${ }^{\mathrm{a}, \mathrm{c} *}$ G. MARÍN ${ }^{\mathrm{b}}$ AND J. WALLMAN" \\ a Programa de Anatomía y Biología del Desarrollo, ICBM, Facultad de, \\ Medicina, Universidad de Chile, Independencia 1027, Santiago, Chile \\ ${ }^{b}$ Laboratorio de Neurobiología, Facultad de Ciencias, Universidad de \\ Chile, Las Palmeras 3425, Santiago, Chile
}

${ }^{c}$ Department of Biology, City College, City University of New York, New York, NY 10031, USA

\begin{abstract}
We studied the stimulus characteristics necessary for the expression of $c$-fos protein in optokinetic system neurons using immunocytochemistry. Using whole-field visual motion as a stimulus, we found substantial $c$-fos expression in the optic tectum ( $\mathrm{TeO}$ ), the nucleus of the basal optic root (nBOR) and the pretectal nucleus lentiformis mesencephali (LM); in all cases immunostaining was seen only on the side contralateral to the eye viewing whole-field unidirectional motion; the side of the brain contralateral to the eye wearing a diffuser showed no staining. In the nBOR and the LM, different regions showed a remarkable specificity of $c$ fos expression depending on the direction of visual motion stimulation. Neurons were stained primarily in regions known from previous electrophysiological recordings to be maximally responsive to that direction of motion; little staining was seen after motion orthogonal to the preferred motion direction. Novel, continuous visual motion stimuli, lasting more than $\mathbf{3 0} \mathrm{min}$, was required for maximal $c$-fos expression, suggesting that brief periods of unidirectional optic flow, as would be experienced during normal life, do not stimulate the expression of $c$-fos. The largest number of neurons was labeled when birds raised from hatching with one eye covered by a diffuser were exposed to full-field visual motion immediately after the diffuser was switched from one eye to the other, so that only the previously naive eye was visually stimulated. We conclude that the expression of $c$-fos in the optokinetic nuclei is linked to near peak firing rates on the one hand, and the novelty and duration of the visual signals, on the other, supporting the assumption that this expression is mainly related to stimulus contexts leading to neuronal plastic changes. (c) 2009 IBRO. Published by Elsevier Ltd. All rights reserved.
\end{abstract}

Key words: accessory optic system, metabolic mapping, neural plasticity, immediate-early genes, chicken.

The immediate-early gene $c$-fos is transiently expressed in neurons immediately after a variety of physiological and pharmacological stimuli (Cole et al., 1989; Morgan and Curran, 1991), and its protein product, like that of other

*Corresponding author. Tel: +56-2-978-6790; fax: +56-2-978-6264. E-mail address: xrojas@med.uchile.cl (X. Rojas).

Abbreviations: AOS, accessory optic system; LM, nucleus lentiformis mesencephali; nBOR, nucleus of the basal optic root; NT, nasal-totemporal; OKN, optokinetic nystagmus; PBST, $0.2 \%$ Triton X-100 in phosphate-buffered saline; TeO, optic tectum; TN, temporal-to-nasal; 2DG, 2-deoxyglucose. well known immediate-early genes such as jun and zif268, functions as a transcription factor, presumably coupling extracellular signals to changes in neuronal function (Morgan and Curran, 1991; Curran and Franza, 1988; Herdegen and Leah, 1998).

Immunocytochemical staining with $c$-fos has been extensively used in mapping functional activity with cellular resolution in a variety of systems (Sagar et al., 1988; Dragunow and Faull, 1989; Montero, 1995; Melzer and Steiner, 1997; Bisler et al., 2002; Illig and Haberly, 2003; Zou et al., 2005; Illig, 2007). Yet, as c-fos expression is linked to neuronal depolarization by a complex signaling cascade (Morgan and Curran, 1986; Sheng et al., 1990; Zhao et al., 2007, see also Gilman et al., 1988 and Thompson et al., 1995), the specific circumstances leading to $c$-fos expression and the consequences of this expression in the normal functioning of neurons remain largely unknown.

To contribute to our understanding of the range of stimuli and contextual factors that produce $c$-fos expression in sensory systems, we have studied the pattern of $c$-fos induction in electrophysiologically well-characterized neurons of the visual system of birds, combining protocols of visual deprivation and highly specific visual stimulation.

The nucleus of the basal optic root (nBOR) of the accessory optic system (AOS) and the pretectal nucleus lentiformis mesencephali (LM) are parts of the visual system that respond best to slow movements of large parts of the visual field in particular directions, as are usually produced by movement of the head. These nuclei (which we will refer to collectively as the optokinetic nuclei) project to several different targets, including the vestibular nuclei and cerebellum and drive stabilizing eye movements such as the optokinetic response (McKenna and Wallman, 1985a; Wylie et al., 2007; Pakan et al., 2006; Pakan and Wylie, 2006). Thus the optokinetic nuclei respond to simple visual stimuli and have a clear function. In birds they have an additional favorable characteristic: Neurons sensitive to particular directions of motion are found together in distinct regions. This has been demonstrated both by electrophysiology (Burns and Wallman, 1981; Wylie and Frost, 1990a,b, 1996; Wylie and Crowder, 2000; Zhang et al., 1999) and by metabolic mapping with 2-deoxyglucose (McKenna and Wallman, 1981, 1985b; Telford and Frost, 1989). Thus, from a neuron's location one can infer, with high probability, its directional tuning. Specifically, nearly all cells recorded in the $\mathrm{nBOR}$ respond to directional visual motion with their peak motion sensitivity clustered around three directions: Upward, downward and nasal-to-temporal (NT). The dorsal region of the nucleus contains mostly 
upward-sensitive neurons and the remainder contains downward-sensitive neurons, with a ventral-most region that contains mostly neurons sensitive to NT motion (Burns and Wallman, 1981; Morgan and Frost, 1981; Wylie and Frost, 1990a). The fourth principal direction, temporal-tonasal (TN) motion, is signaled to by neurons in the LM and by neurons lateral to the nBOR (Burns and Wallman, 1981; Winterson and Brauth, 1985; Fu et al., 1998; Wylie and Frost, 1996; Wylie and Crowder, 2000). In addition, neurons in the LM are responsive to NT motion (in variable proportions, depending on the study) and, to a lesser degree, to vertical visual motion (Fu et al., 1998; Wylie and Crowder, 2000).

Birds also have an essentially crossed optic tract, so that visual responses on one side of the brain are mostly produced by stimulation of the contralateral eye. In the present study, we evaluate $c$-fos expression on both sides of the brain after differential stimulation of the two eyes, and compare the results with those obtained from previous electrophysiological recordings (Burns and Wallman, 1981) and metabolic mapping with 2-deoxyglucose (2DG) (McKenna and Wallman, 1985; Morgan and Frost, 1981).

In brief, we find that (a) in the AOS, LM and superficial layers of the optic tectum ( $\mathrm{TeO})$, the principal visual area in birds, $c$-fos is widely expressed in response to slow, large field visual motion, but not to diffuse light; (b) that this expression is much stronger if the stimulus used is novel and continuous during the period of stimulation; and (c) that within the AOS, the regions showing c-fos immunostaining in response to particular directions of stimulus motion are the same as those shown by electrophysiology to be the peak response direction of those neurons.

\section{EXPERIMENTAL PROCEDURES}

\section{Visual stimulation}

The experiments were carried out using White Leghorn chickens $(n=36)$, between 6 and 12 days of age, and conformed to the guidelines established by the National Institutes of Health Guide for the Care and Use of Laboratory Animals. Every effort was made to minimize the number of animals used and their suffering. Because of the near-complete decussation of the avian visual pathways, in most experiments we exposed one eye to visual motion and covered the other one with a hemispherical white translucent plastic diffuser. This permitted us to compare the $c$-fos immunostaining induced by visual stimulation through a seeing and a diffuser-covered eye. In most experiments the chicks were raised with one eye covered by the diffuser (Wallman et al., 1978). This diffuser essentially eliminated form vision, but attenuated the light by only 0.6 log units, and was either switched to the other eye or removed prior to visual stimulation.

For stimulation with visual motion, birds were placed in a container with the head restrained, and then inside a striped drum rotating at $15 \%$ s. Four directions of visual motion were used as stimuli: upwards, downwards, TN, and NT. For horizontal visual motion stimulation, a vertically striped drum rotated about a vertical axis. For vertical visual stimulation, we used a drum rotating about a horizontal axis, with the walls horizontally striped and one end closed and radially striped, thereby simulating the motion of a sphere. In this arrangement, if both eyes were open and one eye viewed, for example, upward visual motion in the central visual field, the other eye would see a combination of extorsional and downward visual motion (chicken eyes are $130^{\circ}$ apart).

Several experimental protocols were used: in most cases the eye previously covered by a diffuser viewed one direction of motion for $2 \mathrm{~h}$, and the other eye was either covered by a diffuser or open (pooled together and termed diffuser-removed condition, $n=14$ ). In four cases, birds were placed in the drum without removing the diffuser, i.e. the eye stimulated was the previously open one. In addition, six birds had the diffuser switched to the normal eye as before, but they were given only $30 \mathrm{~min}$ of visual motion stimulation, after which three were sacrificed immediately and the other three were kept in darkness for an additional $1.5 \mathrm{~h}$ before sacrifice. Another three birds had the diffuser switched to the other eye and then saw upward visual motion in a discontinuous manner ( $5 \mathrm{~s}$ of visual motion, $25 \mathrm{~s}$ no motion, for $2 \mathrm{~h}$ ). Another four normal, viewing birds were exposed to visual motion either binocularly $(n=2)$, or monocularly $(n=2)$. Six additional birds were returned to the common brooder after removing the diffuser $(n=4)$ or switching it to the other eye $(n=2)$ for $2 \mathrm{~h}$ prior to sacrifice. Table 1 shows these different experimental arrangements.

\section{Immunocytochemistry}

After the stimulation period the birds were perfused with $0.1 \mathrm{M}$ phosphate-buffered saline ( $\mathrm{pH} 7.4)$ followed by $4 \%$ paraformaldehyde in $0.1 \mathrm{M}$ phosphate buffer. After a day or more in fixative, the brains were transferred to $30 \%$ sucrose in phosphate buffer, and subsequently sectioned in the coronal plane at $50 \mu \mathrm{m}$ using a freezing microtome. They were then processed for $c$-fos immunocytochemistry. In brief, the sections were first incubated for $1 \mathrm{~h}$ in $5 \%$ normal goat serum and $0.2 \%$ Triton $\mathrm{X}-100$ in phosphatebuffered saline (PBST), and then incubated with antibodies to $c$-fos in PBST for $48 \mathrm{~h}$, followed by incubation in biotinylated secondary antibody in PBST for $1 \mathrm{~h}$ and subsequently in avidinperoxidase in PBST for $2 \mathrm{~h}$ (ABC kit, Vector Laboratories, Burlingame, CA, USA). The rabbit polyclonal anti-c-fos antibodies (c-fos

Table 1. Summary of the experimental conditions used in the present study

\begin{tabular}{|c|c|c|c|c|c|c|}
\hline \multirow[t]{2}{*}{ Experimental condition } & \multirow[t]{2}{*}{ No. of birds } & \multicolumn{2}{|c|}{ Visual experience } & \multicolumn{2}{|l|}{ Visual motion stimulation } & \multirow[t]{2}{*}{ Stimulus time } \\
\hline & & Eye 1 & Eye 2 & Deprived eye & Viewing & \\
\hline Diffuser removed & 14 & Deprived & Viewing & Up & Deprived & $2 \mathrm{~h}$ \\
\hline Diffuser left in place & 4 & Deprived & Viewing & Deprived & Up or NT & $2 \mathrm{~h}$ \\
\hline \multirow{3}{*}{$\begin{array}{l}\text { Shorter or discontinuous } \\
\text { stimulation }\end{array}$} & 3 & Deprived & Viewing & Up or TN & Deprived & $30^{\prime}$ \\
\hline & 3 & Deprived & Viewing & Up & Deprived & $30^{\prime}+90^{\prime}$ in dark \\
\hline & 2 & Deprived & Viewing & Up & Deprived & $5^{\prime \prime}+25^{\prime \prime}$ for $2 \mathrm{~h}$ \\
\hline No occlusion & 4 & Viewing & Viewing & Up, down or $\mathrm{TN}^{*}$ & Deprived or Open* & $2 \mathrm{~h}$ \\
\hline Control stimulation & 6 & Deprived & Viewing & Non-specific stimulation in brooder & Deprived or Open & $2 \mathrm{~h}$ \\
\hline
\end{tabular}

* In these cases, the different stimuli are assigned randomly to one eye or the other, since no eye was occluded. 
689/5), which were a gift from Dr. Rodrigo Bravo (Bristol-Myers Squibb Pharmaceutical Research Institute), had been raised against the whole $c$-fos protein, and have been described previously (Kovary and Bravo, 1991; Leah et al., 1991). They were used at a dilution of 1:5000. Finally, the sections were reacted with diaminobenzidine (Sigma-Aldrich, St. Louis, MO, USA) and peroxide plus $\mathrm{N}_{2} \mathrm{SO}_{4} / \mathrm{CoS}_{2}$, mounted, dehydrated and coverslipped.

\section{Quantification of c-fos-stained neurons}

To compare the number of stained neurons in different regions of the nBOR, we selected six transverse sections (two rostral, two middle, and two caudal; at approximately $170 \mu \mathrm{m}$ intervals), which represent approximately one-fourth of the sections, and, with the aid of a camera lucida, we drew the outline of each selected section and the location of all stained neurons. We then superimposed to each drawing the appropriate template of the outline of the rostral, middle or caudal nucleus, drawn from thionine stained sections. Each template was divided in four quadrants, and the stained nuclei in each quadrant were counted. These numbers apply only to the sections drawn and thus underestimate the number of stained neurons in the entire nucleus by a factor of about four. In the $\mathrm{LM}$ and $\mathrm{TeO}$, we made a qualitative fourcategory assessment of the immunostaining $(-,+,++$ and +++ ).

\section{RESULTS}

\section{General findings}

One of our principal findings is that novel, continuous visual motion stimulation was required for strong $c$-fos expression in the optokinetic nuclei. Unidirectional visual motion strongly induced $c$-fos expression in neurons receiving input from the viewing eye (i.e. on the contralateral side of the brain) in different parts of the nBOR (Fig. 1A, B) and pretectum (LM; Fig. 1E, F), depending on the direction of visual motion used as stimulus. We also found $c$-fos expression in the $\mathrm{TeO}$, the main visual area of birds (Fig. $1 \mathrm{C}, \mathrm{D})$. In some brains, visual motion stimulation also resulted in staining in the nucleus geniculatus pars ventralis (GLv) and in the putative avian equivalent to the suprachiasmatic nucleus (the lateral retinorecipient region of the hypothalamus), areas that have been shown to respond to whole-field directional visual motion (Hossokawa, 1996; Wallman et al., 1994). No neurons in any visual area showed $c$-fos expression contralateral to the eye covered by a diffuser (Fig. 1A, C, E), indicating that the basal level of $c$-fos expression in the visual system is so low that it is undetectable by immunocytochemistry. However non-visual areas in the hypothalamus and the central gray of the mesencephalon showed consistent bilateral $c$-fos staining in all birds. The nucleus of the dorsal lateral lemniscus was also bilaterally stained in most brains.

Three factors consistently influenced the degree of c-fos expression at any location: the direction of stimulus motion, the duration of stimulation, and the novelty of the stimulation compared to the animal's previous visual experience.

\section{Stimulus motion direction}

Unidirectional visual motion stimulation resulted in the strongest immunostaining in the optokinetic nuclei, with the c-fos expressing nuclei found in different regions according to the direction of visual motion used as a stimulus. This parcellation of the stained nuclei was most prominent in the AOS.

$n B O R$. In the $\mathrm{nBOR}$, quite distinct regions expressed $c$-fos depending on whether the visual stimulation was upward, downward or NT motion. Fig. 2 summarizes the distribution of $c$-fos expression in the rostral, middle and caudal thirds of the nucleus, each of which was divided into quadrants. In this figure, we summed all neurons contralateral to a previously deprived eye subsequently exposed to visual motion. In all but two birds the diffuser was switched (the two birds that had the diffuser removed are marked with an asterisk in Table 2). Approximately half of the neurons expressing $c$-fos were located in the middle third, where the nucleus is larger.

Cells expressing c-fos after upward visual motion stimulation are found in the rostral third of the nBOR and in the dorsal region of the remainder (Fig. 2). Cells expressing c-fos after downward visual stimulation were located throughout the caudal two-thirds of the nucleus, with the highest density of stained nuclei found in the ventral medial quadrant (Fig. 2, middle panel). Finally, most NT visual motion-induced stained cells were located in the ventral region of the caudal two-thirds of the $\mathrm{nBOR}$; more than $50 \%$ of these cells were found in the ventral lateral quadrant (Fig. 2, lower panels). When we organized the data from Fig. 2 into seven categories (three rostral-caudal division, two dorso-ventral divisions, two mediolateral divisions), we found that all birds but one in each group (upward motion group, downward motion group and NT motion group) showed a significant parcellation of immunostaining among these categories, with 9 of the 12 birds showing highly significant parcellation $\left(P<0.001, \chi^{2}>14\right.$, $d f=3)$.

Based on these general findings, we quantified the pattern of $c$-fos staining by reclassifying the stained cell counts into four regions: the rostral third as a whole (R), with the remainder of the nucleus (middle and caudal $\mathrm{nBOR}$ ) divided into the dorsal half (D), the ventral medial quadrant (VM) and the ventral lateral quadrant (VL). By summing all the cells found in each of these regions in each experimental group (Table 2), we found that the distribution of cells among these three groups differed in each of the four regions in which we divided the data $\left(P<0.001, \chi^{2}=1232, d f=6\right)$. This is also true if we examine only the up- and down-groups in the first two regions, or only the down- and nasotemporal groups in the last two regions (in both cases $P<0.001 ; \chi^{2}>167, d f=1$ ).

As the cell counts in Table 2 indicate, the rostral third of the nBOR showed the strongest specificity for upward visual motion stimulation; in this region $81.8 \%$ of all $c$-fos expressing cells were stained after upward visual motion stimulation, with an average of 115.4 stained nuclei for upward and 25.5 for all other directions combined. Throughout the nucleus, up-responding cells were located dorsally, especially in the middle third (Fig. 3B), with a dorsal-to-ventral ratio of 3.0 for the whole nucleus (Fig. 3B, 

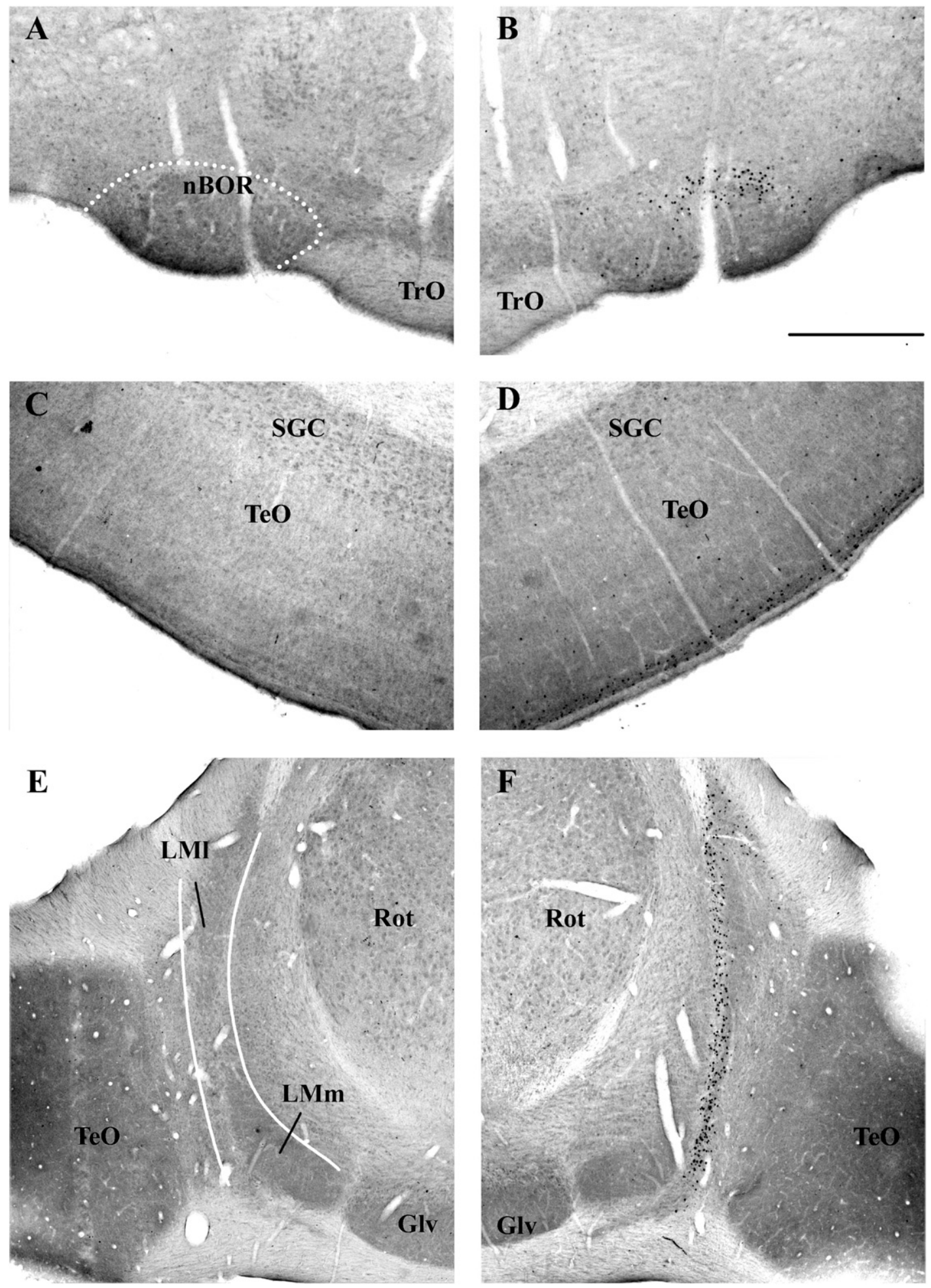

Fig. 1. Photomicrographs of coronal sections of the chick brain at different midbrain levels, showing c-fos expression on the $n B O R(B)$, TeO (D), and LM area (F) contralateral to the eye stimulated with whole-field visual motion for $2 \mathrm{~h}$. In these birds there was no $c$-fos expression in the visual nuclei contralateral to the eye covered by a diffuser (A, C, E). In F the LM area is outlined in white. Glv: nucleus geniculatus pars ventralis; LMm: nucleus lentiformis mesencephali, pars medialis; LMI: nucleus lentiformis mesencephali, pars lateralis; TrO: optic tract; Rot: nucleus rotundus; SGC: stratum griseum et fibrosum superficiale; Scale bar $=500 \mu \mathrm{m}$.

Table 2). This localization was especially prominent in the dorsal medial quadrant of the central nBOR, where 5.2 times as many cells were stained as in the ventral medial quadrant. 


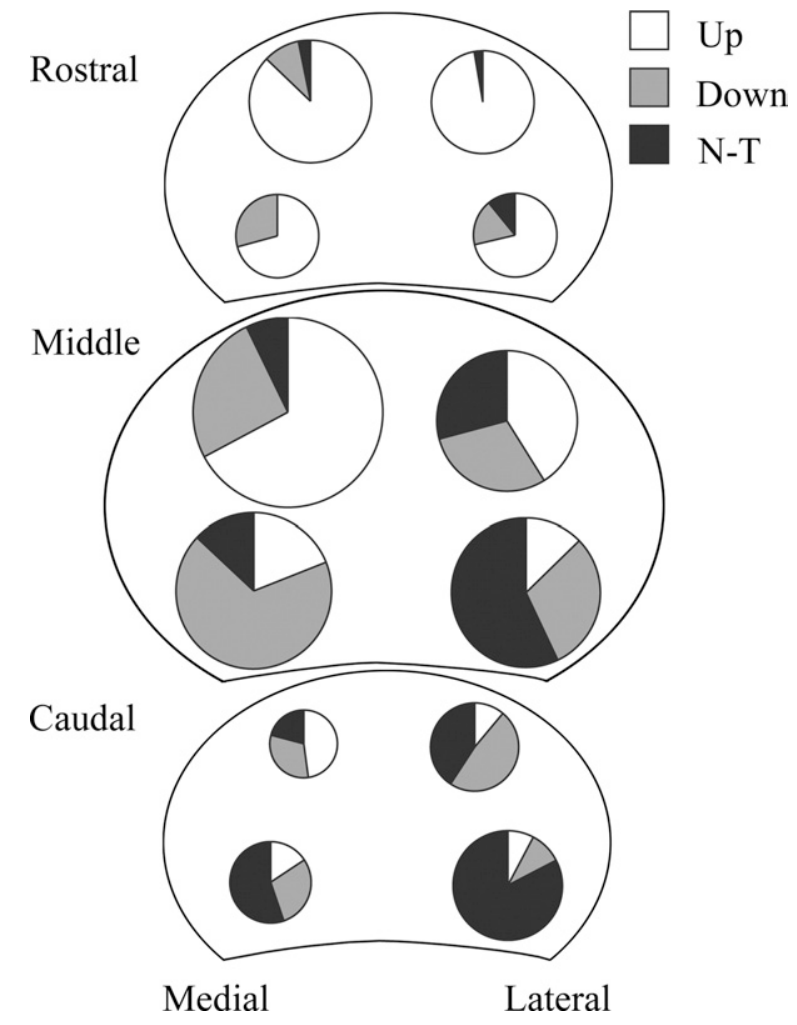

Fig. 2. Schematic that summarizes the distribution of $c$-fos immunoreactive cells found in the $\mathrm{nBOR}$ of 12 birds after visual stimulation of the contralateral (previously deprived) eye. Each panel represents a transverse section through the rostral, middle or caudal nBOR. The pie charts show the total number of $c$-fos expressing cells after visual stimulation with upward (up), downward (down) and backward (NT) in the four quadrants in which we divided the nucleus: dorsal medial, dorsal lateral (top row in each panel), ventral medial and ventral lateral (bottom row in each panel). The area of each pie indicates the number of labeled cells, ranging from 24 for the ventral lateral quadrant in the rostral nBOR, to 129 for the dorsal medial quadrant in the middle $\mathrm{nBOR}$.

The pattern of $c$-fos expression in the nBOR after downward and NT visual motion was somewhat complementary to that obtained after upward visual motion, in that there were few stained cells in the rostral third of the nucleus and in the dorsalmost cap, and many stained cells in the caudal third. Thus, the ratio of cells stained in the caudal vs. rostral thirds after downward visual motion was 6.37 , almost complementary to the rostral/caudal ratio of 6.6 seen after upward visual motion. For NT motion, the caudal/rostral ratio was even larger, 12.3, mainly accounted for by the relative absence of rostrally located cells responsive to horizontal motion.

Cells expressing c-fos after downward visual motion stimulation were located throughout the dorsoventral extent of the nucleus, except for the dorsalmost region, with a slight ventral-dorsal parcellation in the middle and caudal nBOR (V/D ratio=1.3) (Fig. 3B, Table 2). Although this ratio suggests that the dorsal-ventral division between up-responsive and down-responsive cells is less extreme than shown by electrophysiology (Burns and Wallman, 1981; Wylie and Frost, 1990a), Fig. 3 shows that the actual location of the upward motion induced c-fos expressing cells was a band located in the dorsalmost cap of the nucleus (Fig. 3B), whereas there are almost no down cells in this region (Fig. 3D). This clear separation became diluted in our numerical data by dividing the nucleus in dorsal and ventral halves.

NT visual motion resulted in c-fos-stained cells located ventrally and laterally, with a ventral/dorsal ratio of 2.35 , and a lateral/medial ratio of 2.99. Overall, of all cells stained after NT visual motion stimulation, 54\% were located in the ventral lateral quadrant (Fig. 3F, Table 2).

TN visual motion stimulation resulted in virtually no c-fos expression in the nBOR. On average, TN cells represent only $3.5 \%$ of all cells stained ( 26.5 cells out of an average for all directions combined of 764.4 cells), but in heavy staining in a small area lateral to the AOS (Fig. 3G), called the lateral nBOR (nBORI) by McKenna and Wallman (1985) and by Bodnarenko et al. (1988), who showed by the 2DG method that it responded to TN visual motion. This region of stained cells could be traced rostrally until it fused with the ventralmost part of the LM.

Finally, purely extorsional (clockwise direction of motion to the right eye) visual motion stimulation resulted in a different pattern of distribution of stained nuclei: cells were located along the boundaries of the nBOR, forming a thin semicircular band that spanned the dorsomedial and ventromedial borders of the nucleus (Fig. 4).

LM. In the LM, the other optokinetic nucleus in the avian midbrain, the strongest $c$-fos expression was seen after TN visual motion stimulation, but we also found stained nuclei after NT motion and, in two out of four cases, after downward motion. The LM is divided in two apposed subnuclei whose boundaries and nomenclature differ depending on the authors (Kuhlenbeck, 1939; Ehrlich and Mark, 1984; Bodnarenko et al., 1988; Gamlin and Cohen, 1988). In the Gamlin and Cohen (1988) nomenclature followed here, these subnuclei are called the medial and lateral LM.

TN motion resulted in a narrow dorsoventral strip of stained nuclei located mostly along the border between the medial and lateral divisions of the LM (Fig. 5A, B), this band of stained cells extended from the rostral to the caudal tip of the nucleus, where it merged with the lateral part of the nBOR (Fig. 3G). In contrast, NT motion resulted in stained nuclei located mostly in the caudal half of the LM, including its lateral division (Fig. 5C, D), although the divisions are hard to define in our material. These findings are consistent with those reported for electrophysiological recordings, which show that most cells in the LM prefer horizontal visual motion, although there is a disagreement in the literature about the proportion of cells responsive to each direction (Winterson and Brauth, 1985; Wylie and Crowder, 2000; Fu et al., 1998). It is generally accepted that the LM is homologous to the nucleus of the optic tract (NOT) of mammals, which contains many cells with this directional preference and plays a major role in TN optokinetic responses (Wallman, 1993). 
Table 2. Cell counts from birds that had the previously deprived eye exposed to visual motion stimulation for $2 \mathrm{~h}$

\begin{tabular}{|c|c|c|c|c|c|c|c|c|}
\hline \multirow[t]{2}{*}{ Exp. condition } & \multirow[t]{2}{*}{ Bird no. } & \multirow[t]{2}{*}{ Rostral } & \multicolumn{3}{|c|}{ Middle and caudal } & \multirow[t]{2}{*}{ Total } & \multirow[t]{2}{*}{$\mathrm{TeO}$} & \multirow[t]{2}{*}{ LM } \\
\hline & & & Dorsal $(\mathrm{M}+\mathrm{L})$ & Ventromedial & Ventrolateral & & & \\
\hline \multirow[t]{8}{*}{ Up } & 98 & 222 & 265 & 40 & 24 & 551 & $++^{* *}$ & - \\
\hline & 99 & 16 & 14 & 0 & 1 & 31 & ++ & - \\
\hline & 145 & 123 & 101 & 16 & 21 & 261 & ++ & - \\
\hline & 144 & 141 & 105 & 23 & 6 & 275 & ++ & - \\
\hline & 149 & 75 & 129 & 19 & 13 & 236 & ++ & - \\
\hline & Mean & 115.4 & 122.8 & 19.6 & 13 & & & \\
\hline & S.D. & 76.79 & 90.68 & 14.36 & 9.72 & & & \\
\hline & $\%$ & $42.6 \%$ & $45.3 \%$ & $7.2 \%$ & $4.8 \%$ & $100 \%$ & & \\
\hline \multirow[t]{7}{*}{ Down } & 118 & 11 & 112 & 100 & 8 & 231 & ++ & + \\
\hline & 114 & 31 & 46 & 70 & 41 & 188 & ++ & - \\
\hline & 6 & 25 & 303 & 266 & 138 & 732 & ++ & + \\
\hline & $102^{*}$ & 8 & 53 & 22 & 33 & 116 & + & - \\
\hline & Mean & 18.75 & 128.5 & 114.5 & 55 & & & \\
\hline & S.D. & 11.03 & 120.04 & 105.99 & 57.09 & & & \\
\hline & $\%$ & $5.9 \%$ & $40.5 \%$ & $36.1 \%$ & $17.4 \%$ & $100 \%$ & & \\
\hline \multirow[t]{6}{*}{ NT } & 104 & 6 & 48 & 36 & 139 & 229 & ++ & ++ \\
\hline & 152 & 3 & 6 & 5 & 8 & 22 & + & + \\
\hline & $106^{*}$ & 6 & 76 & 30 & 88 & 200 & +++ & +++ \\
\hline & Mean & 5 & 43.3 & 23.7 & 78.3 & & & \\
\hline & S.D. & 1.73 & 35.23 & 16.44 & 66.03 & & & \\
\hline & $\%$ & $3.3 \%$ & $28.8 \%$ & $15.7 \%$ & $52.1 \%$ & $100 \%$ & & \\
\hline \multirow[t]{3}{*}{$\mathrm{TN}$} & 107 & 8 & 12 & 0 & 6 & 26 & ++ & +++ \\
\hline & 148 & 2 & 7 & 7 & 11 & 27 & +++ & +++ \\
\hline & Mean & 5 & 9.5 & 3.5 & 8.5 & & & \\
\hline
\end{tabular}

6 sections per bird ( 2 each for rostral, middle and caudal nBOR) were counted.

* In these birds, the diffuser was removed prior to visual motion stimulation. The data shown is from the side of the brain contralateral to the previously deprived eye.

${ }^{* *}$ In the LM and TeO, a qualitative 4-category assessment of the immunostaining was made $(-,+,++$ and +++$)$.

TeO. In the TeO, prolonged unidirectional visual motion stimulation in any of the four directions of visual motion we used consistently resulted in numerous nuclei expressing c-fos with no apparent spatial segregation of the stained cells by direction of motion stimulation. Birds that had the diffuser switched to the previously open eye before being stimulated with whole-field visual motion in any direction, showed c-fos-expressing-nuclei confined to the superficial, retinorecipient layers of the tectum (stratum griseum and fibrosum superficialis), mainly in layers 2-4 and 6 (Fig. 6B).

In contrast, birds that were returned to the common brooder for $2 \mathrm{~h}$ after switching the diffuser to the previously open eye, thus giving them visual stimulation that was novel but not unidirectional, showed most tectal $c$-fos expression in deeper layers, with a large concentration of stained nuclei in layers 10 and 13 (Fig. 6C), which were usually unstained after stimulation with whole-field directional motion. Tectal $c$-fos expression in these birds was most striking in the regions that receive input from the inferior nasal and superior temporal retina. In contrast, the optokinetic nuclei of these birds that were returned to the cage after switching the diffuser to the previously open eye showed, on average, fewer stained nuclei than the visual motion group (128 vs. 256 per bird), and the neurons expressing $c$-fos were distributed all over the nBOR, with no apparent localization.

\section{Duration of stimulus}

In the experiments discussed above, strong $c$-fos expression generally required a long period of visual stimulation. Shorter periods of stimulation, between 75 and $120 \mathrm{~min}$ produced $c$-fos staining equal in intensity and extent. Contrary to our expectations, the limiting factor seemed not to be the length of time available after the stimulus, during which the neurons could accumulate enough of the $c$-fos protein to be detectable by immunocytochemistry. In three birds, 30 min of stimulation followed by $90 \mathrm{~min}$ in the dark before sacrifice resulted in very few stained nuclei (Table 3). Furthermore, in two birds that were stimulated for $2 \mathrm{~h}$ with upward visual motion in a discontinuous manner (the drum rotated for $5 \mathrm{~s}$ and then stopped for $25 \mathrm{~s}$, thus providing a total of $20 \mathrm{~min}$ of stimulation), very little $c$-fos immunostaining was apparent (Table 3 ).

Curiously, in three birds that were exposed to visual motion stimulation for 30 min with no extra time allowed, there were on average more stained cells than in the two cases just described, despite having had less time for protein to build up (Table 3 ). These results are considered further in the Discussion. 

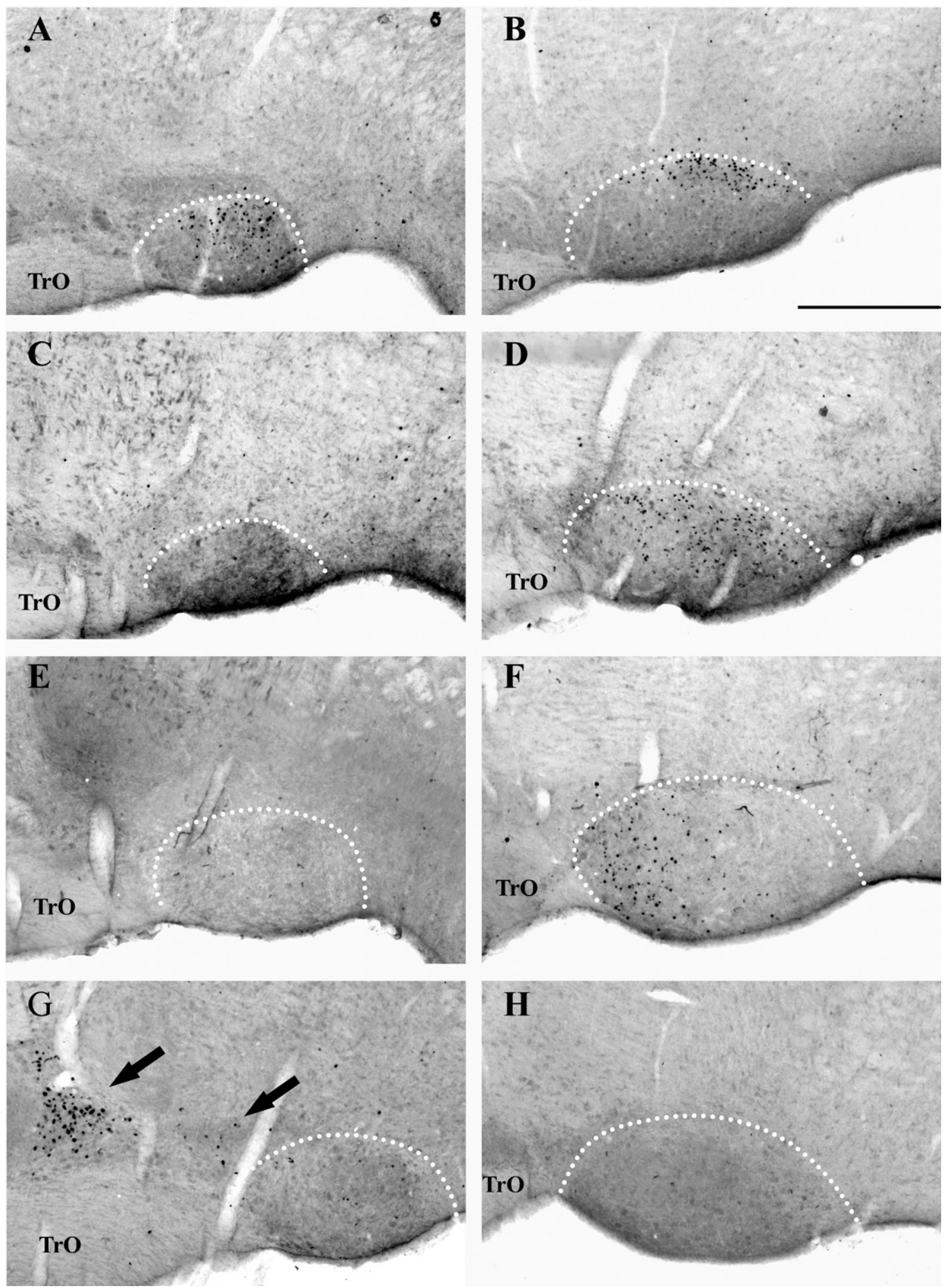

Fig. 3. Photomicrographs of coronal sections of chick brains at the level of the rostral (left panels) and middle (right panels, B, D, F) or caudal (right panels, H) nBOR, showing the distribution of $c$-fos immunoreactive cells after visual motion stimulation in four directions. Note that upward visual motion stimulation resulted in $c$-fos expression in the rostral third of the nucleus and in the dorsal region of the remainder (A, B). Downward visual motion stimulation expressed $c$-fos mainly in the ventral medial region of the middle and caudal nBOR (C, D). NT visual motion stimulation expressed $c$-fos mostly in the ventral lateral region of middle and caudal $n B O R(E, F)$. TN visual motion expressed $c$-fos in a small area lateral to the $n B O R(G, H)$. Scale bar $=500 \mu \mathrm{m}$. 

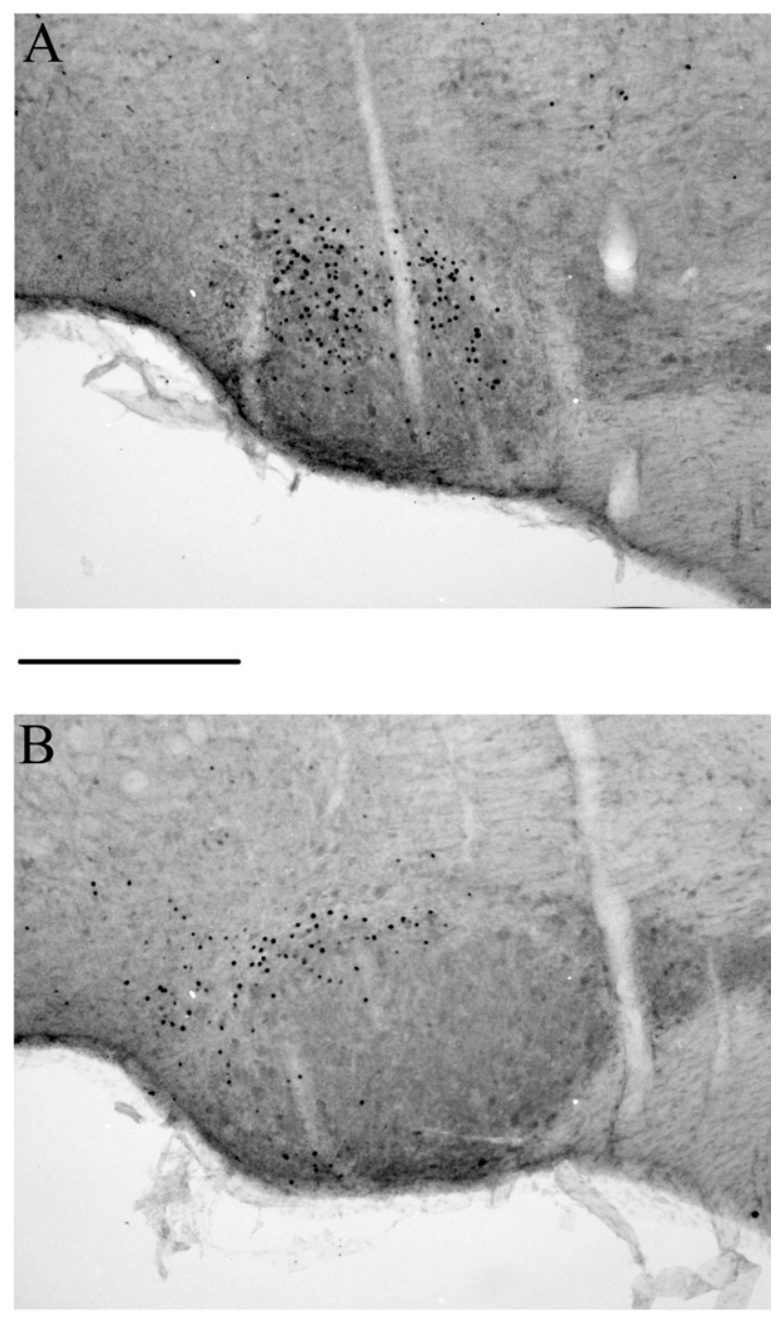

Fig. 4. Photomicrographs of coronal sections of chick brains at the level of the nBOR, showing $c$-fos immunoreactive cells after visual motion stimulation in the extorsional (excyclorotation) direction to the previously deprived eye. Labeled cells are found in the dorsal and medial $n B O R$ at rostral levels $(A)$, and along the nucleus boundaries in the middle $\mathrm{nBOR}(\mathrm{B})$. Scale bar $=500 \mu \mathrm{m}$.

\section{Effect of prior visual deprivation}

In the nBOR, we find that the largest number of labeled cells was obtained when the diffuser was either switched or removed, and the previously deprived eye was stimulated with continuous motion.

The second highest number of stained cells was obtained in three nondeprived birds that were binocularly or monocularly stimulated with unidirectional visual motion. Although the total number of cells in the nBOR of these birds was lower than that of the deprived group (155 vs. 256 ), there is great variability in the individual numbers (Table 3).

The least number of stained cells was found in four cases in which we left the diffuser in place and stimulated the viewing eye of monocularly deprived birds with unidirectional visual stimulation. In these birds, only $7.3 \%$ as many cells ( 14 vs. 256 ) were stained in the nBOR as in the birds with the diffusers switched or removed before unidi- rectional visual stimulation (Table 3). We find it intriguing that these results differ so much from the ones reported in the above paragraph (14 vs. 155 stained cells, on average), considering that in both of these cases the stimulated eye had the same history and experience, and they only differed in the condition of the fellow eye. In the Discussion, we suggest a possible explanation for these results.

Interestingly, binocular stimulation consistently resulted in c-fos expressing cells in the ventral tegmental area, located just dorsal to the nBOR. This area has been shown by electrophysiology to contain binocular cells (Wylie and Frost, 1990b) and to project to the medial column of the inferior olive, which is involved in optic flow analysis (Wylie, 2001).

In the LM there was no obvious difference in c-fos expression in response to TN motion between birds that had the previously deprived eye or the previously viewing eye exposed to visual motion (compare Fig. $1 \mathrm{~F}, 1 \mathrm{E}$ ), suggesting that not all visual system nuclei require prior deprivation for $c$-fos expression.

In the $\mathrm{TeO}$, a somewhat simpler effect of novelty was observed: Similar to the nBOR, there was no $c$-fos expression in the tectum of birds in which the previously viewing eye was stimulated (the diffuser was left in place), although we saw numerous stained nuclei in birds in which the previously covered eye was stimulated (diffuser switched before stimulation) (Tables 2 and 3,c-fos expression in $\mathrm{TeO}$ ). In three cases in which the diffuser was removed and thus both eyes were stimulated with visual motion, the tectum contralateral to the eye that had always been viewing showed somewhat less $c$-fos expression than the tectum contralateral to the previously deprived eye, and in general lacked the thin row of stained nuclei in layer 6 (Fig. $6 \mathrm{~A}$; compare with more widely distributed stained cells in the superficial layers of the tectum in Fig. 6B).

As mentioned above, switching the diffuser to the previously open eye for $2 \mathrm{~h}$ and then returning the birds to the common brooder for $2 \mathrm{~h}$ resulted in numerous c-fosstained nuclei in the $\mathrm{TeO}$. In contrast, in four birds in which the diffusers were simply removed before being returned to their cage for $2 \mathrm{~h}$ we saw few stained nuclei on the side of the brain contralateral to the previously deprived eye, and almost no $c$-fos expression on the side of the brain contralateral to the continuously open eye (Table 3 ). Overall, these results indicate that in the $\mathrm{TeO}$, the largest number of stained cells is seen when the continuously deprived eye was stimulated and the smallest number in those in which the previously open eye was stimulated, suggesting a strong novelty requirement for $c$-fos expression.

\section{DISCUSSION}

The present study sought to characterize the adequate stimulus for $c$-fos expression in optokinetic nuclei neurons. We find that: (a) directional, visual motion stimulation induces $c$-fos expression in different regions of the $\mathrm{nBOR}$ and LM depending on the motion direction of the visual stimuli, and (b) both continuous visual motion stimulation 

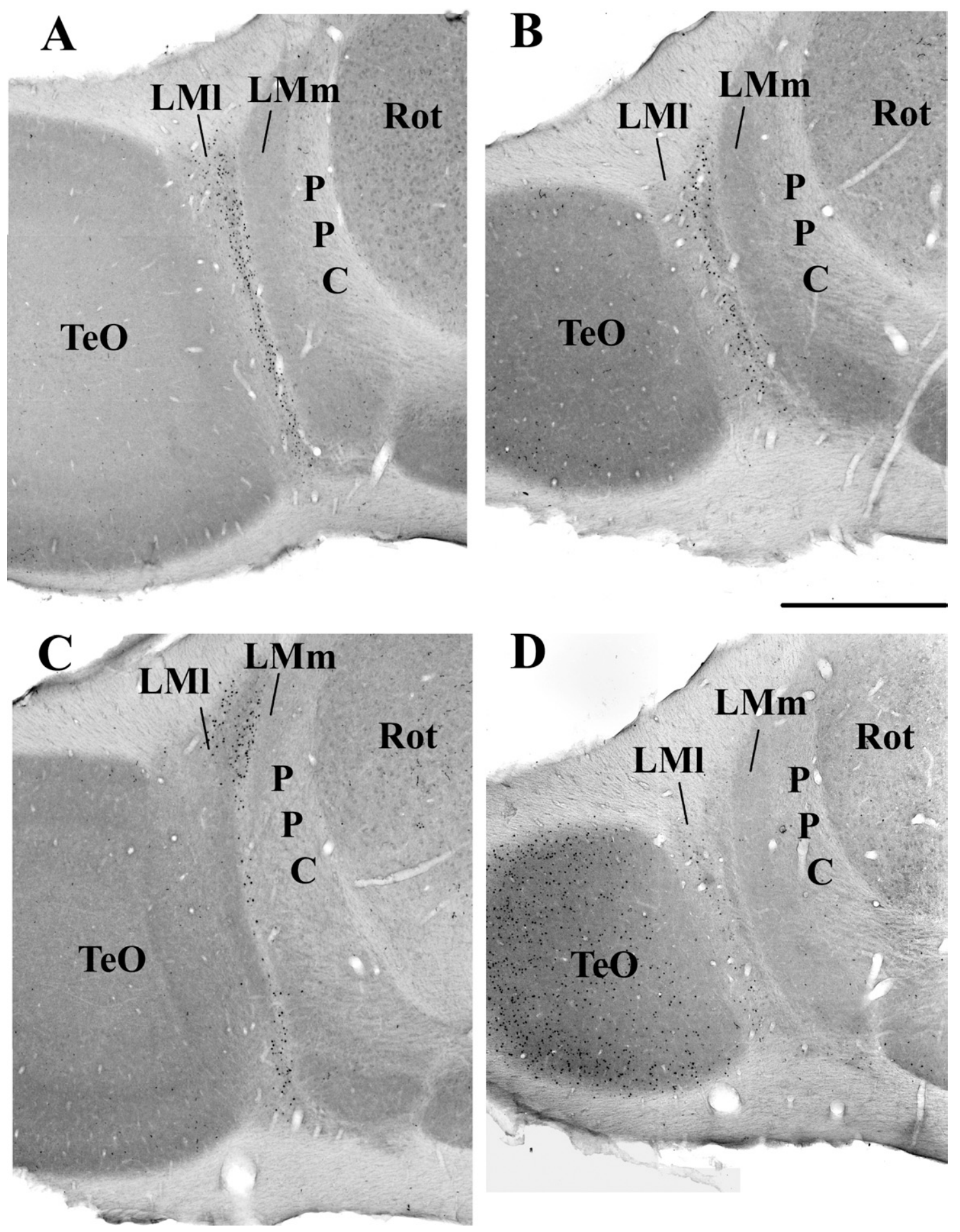

Fig. 5. Photomicrographs of coronal sections of chick brains at the level of the LM, showing $c$-fos immunoreactive cells after horizontal visual motion stimulation to the previously deprived eye. (A, B) TN direction, and (C, D) NT direction. Sections are through the caudal half (A, C) and rostral half $(B, D)$ of the nucleus. Labeled cells appear in LMI ( $D$ and superior part in $C)$, and along the boundary between LMl and LMm ( $A-C)$. Scale bar=1 mm.

and novelty of the stimulus are important determinants of the degree of $c$-fos expression in these nuclei.

In contrast, in the $\mathrm{TeO}$, the main visual area of birds, continuous, novel visual motion stimulation induces c-fos expression in a small subset of neurons, with no obvious seg- regation by stimulus direction. However, non-specific, novel visual stimulation as experienced by a previously covered eye in the common brooder, resulted in more extensive, in some cases massive, tectal c-fos expression, in contrast to the weak labeling this produced in the optokinetic nuclei. 

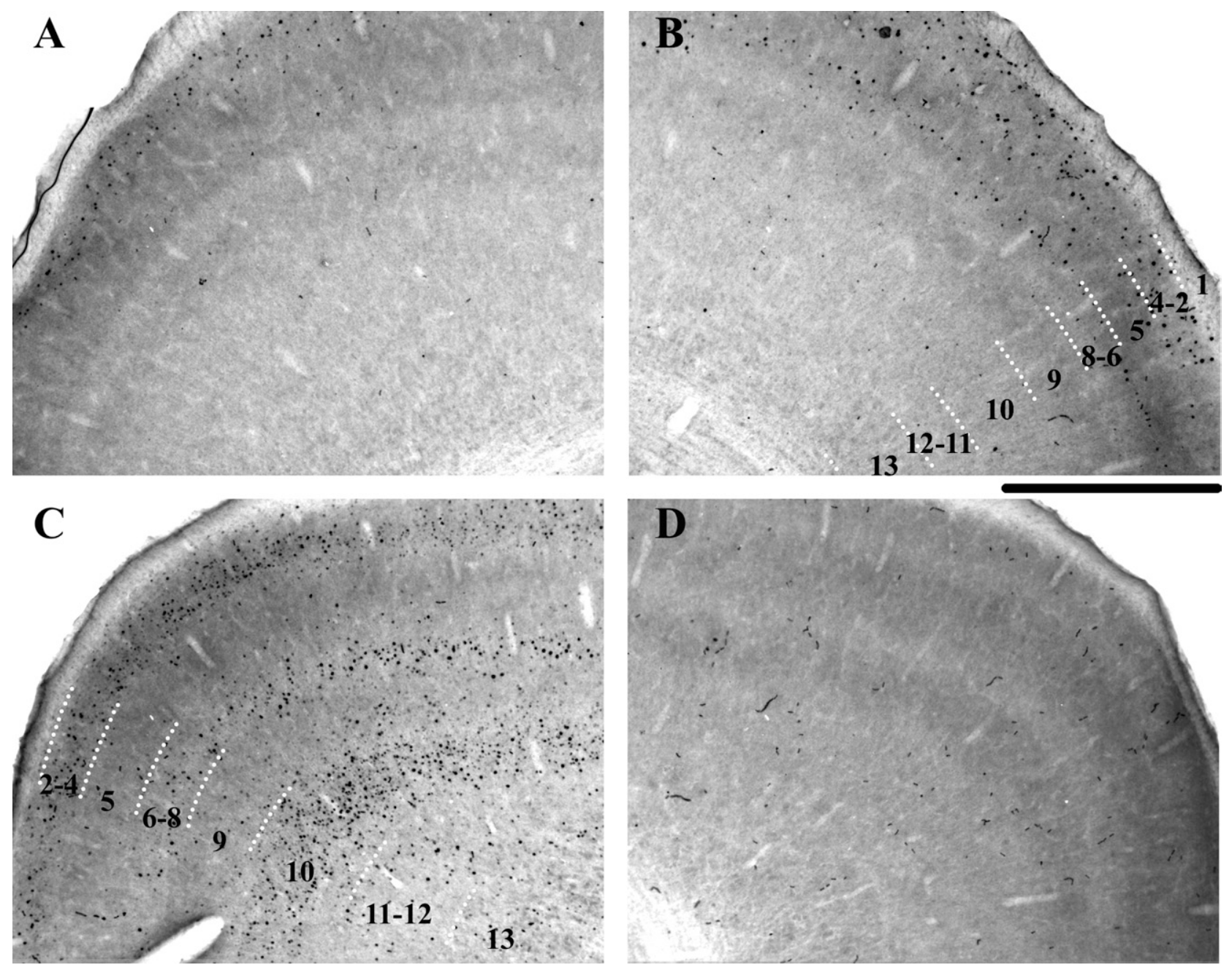

Fig. 6. Photomicrographs of coronal sections of chick brains at the level of the $\mathrm{TeO}$, showing $c$-fos immunoreactive cells contralateral to the previously closed eye after visual motion stimulation (A) in the TN and (B) in the NT directions. (C, D) Massive $c$-fos expression in a bird that was returned to the brooder after removing the diffuser. (C) Tectum contralateral to the previously covered eye. (D) Tectum contralateral to the previously open eye. Numbers indicate tectal layers. Scale bar $=500 \mu \mathrm{m}$.

\section{Directional parcellation of optokinetic nuclei shown by $c$-fos expression compared to other methods}

The spatial pattern of $c$-fos expression in the nBOR and $L M$ reported here is in general agreement with that shown by electrophysiological and 2DG studies, but affords a more precise delineation of the functional divisions of these nuclei than is possible with the other methods. In the $\mathrm{nBOR}$, we find that the pattern of distribution of $c$-fos expressing cells by stimulus motion direction is different for the rostral region compared to the middle and caudal regions. In the rostral $\mathrm{nBOR}$, the vast majority ( $82 \%)$ of all $c$-fos expressing cells is up-sensitive, whereas in the middle and caudal nBOR, up-neurons are located preferentially in the dorsal region of the nucleus, with down-sensitive cells located mainly in the ventral medial region, and NT cells located mostly in the ventral lateral region of the middle and caudal nBOR. The finding that up-sensitive cells are located in the dorsal part of the caudal two-thirds of the nucleus is in agreement with the findings of the 2DG and electrophysiological studies, as is the finding that nasal to temporal cells tend to be located ventrally in the nucleus (Morgan and Frost, 1981; Burns and Wallman, 1981; Natal et al., 1989; McKenna and Wallman, 1985; Wylie and Frost, 1990; Zhang et al., 1999). The fact that the rostral third of the nBOR responds almost exclusively to upward visual motion has not been reported before. However, in the only study that reports recordings from the whole rostral-caudal extent of the nBOR (Wylie and Frost, 1990a), the authors depict a higher percentage of upward sensitive cells in the rostral nBOR (see Fig. 4 in that reference). The further segregation of down and NT-sensitive cells along the medial-lateral axis of the middle $\mathrm{nBOR}$, has not been reported before (but again, see Fig. 4 in Wylie and Frost (1990a). We assume that this is because in most electrophysiological experiments the medial and lateral edges of this small nucleus were not sampled as thoroughly as the center, and thus any difference in the distribution of neurons along the medial lateral axis would 
Table 3. Counts of $c$-fos labeled cells from birds exposed to visual motion stimulation under various conditions. The nBOR counts are based on 6 sections per bird

\begin{tabular}{|c|c|c|c|c|c|c|}
\hline Exp. condition & Bird no. & $\begin{array}{l}\text { Total no. of labeled } \\
\text { cells in nBOR }\end{array}$ & Mean & SD & $\begin{array}{l}\text { c-fos expression } \\
\text { in } \mathrm{TeO}\end{array}$ & $\begin{array}{l}\text { c-fos expression } \\
\text { in LM }\end{array}$ \\
\hline \multicolumn{7}{|l|}{ Previously occluded eye stimulated with } \\
\hline $2 \mathrm{~h}$ of visual motion (up, down and NT) & See Table 2 & See Table 2 & 256.0 & 201.9 & See Table 2 & See Table 2 \\
\hline \multirow{4}{*}{$30^{\prime}$ of visual motion (up or down) } & & & 58.3 & 19.1 & & \\
\hline & 137 & 64 & & & - & - \\
\hline & 140 & 37 & & & - & - \\
\hline & 141 & 74 & & & + & - \\
\hline \multirow[t]{4}{*}{$30^{\prime} U p+90^{\prime}$ in dark } & & & 8.7 & 10.3 & & \\
\hline & 97 & 0 & & & - & - \\
\hline & 95 & 6 & & & - & - \\
\hline & 96 & 20 & & & - & - \\
\hline \multirow[t]{3}{*}{$5^{\prime \prime} U p+25^{\prime \prime}$ no motion } & & & 21.5 & & & \\
\hline & 129 & 33 & & & - & - \\
\hline & 126 & 10 & & & - & - \\
\hline \multirow[t]{3}{*}{ Non-specific visual stimulation } & & & 128.5 & & & \\
\hline & 103 & 192 & & & +++ & + \\
\hline & 117 & 65 & & & ++ & + \\
\hline Continuously open eye stimulated with & & & 18.75 & 5.3 & & \\
\hline \multirow[t]{3}{*}{2 h Up } & 119 & 23 & & & - & - \\
\hline & 120 & 11 & & & - & - \\
\hline & 139 & 21 & & & - & - \\
\hline $2 \mathrm{~h} \mathrm{NT}$ & 151 & 20 & & & + & - \\
\hline No occlusion & & & 146 & 90.2 & & \\
\hline $2 \mathrm{~h}$ binocular stim. Up & 101 & 152 & & & + & - \\
\hline Down & 110 & 154 & & & + & - \\
\hline $2 \mathrm{~h}$ monocular stim. Down & 109 & 249 & & & + & - \\
\hline TN & 105 & 29 & & & ++ & ++ \\
\hline
\end{tabular}

have been overlooked. Consequently, c-fos immunocytochemistry offers a better picture of the topography of neurons responsive to visual motion.

We also found that clockwise visual stimulation resulted in a different pattern of label from that of the four previous directions of visual motion used as stimuli, which may reflect cells that respond maximally to pure torsional monocular stimulation. Wylie and Frost (1999) found a subpopulation of binocular cells in the nBOR of pigeons that preferred rotational optic flow, but in those cells upward and downward preferences were located in opposite visual fields. Another possibility is that this label pattern could be obtained by activating a subset of upward sensitive neurons responding to visual motion in the frontal visual field and a subset of downward sensitive neurons responding to visual motion in the posterior visual field.

c-fos immunochemistry, electrophysiology, and 2DG do not show TN sensitive neurons in the nBOR, and all three demonstrate a population of $\mathrm{TN}$ responsive cells lateral to the $\mathrm{nBOR}$. Both the 2DG and c-fos methods show that these TN responsive cells can be traced rostrally to the LM. In this nucleus, electrophysiology (Winterson and Brauth, 1985; Fu et al., 1998; Wylie and Crowder, 2000) and 2DG studies (McKenna and Wallman, 1985; Bodnarenko et al., 1988) have shown that many neurons (up to $53 \%$, in some reports) respond to $\mathrm{TN}$ visual motion stimulation, with smaller populations responding to NT, downward and upward visual motion. Our findings are in agreement with this, except for the fact that we did not see any c-fos-stained cells after upward visual motion stimulation, perhaps because we did not study the rostralmost part of the LM in these birds. Alternatively, the stimulus speed used in this study $\left(15^{\circ} / \mathrm{s}\right)$ may have not been optimal to activate upward sensitive cells in the LM, which seem to respond to very fast stimuli (see Fig. 5A in Wylie and Crowder, 2000).

Overall, our findings indicate that in the optokinetic nuclei (nBOR and LM), c-fos expression after novel visual motion in a particular direction is indicative of that neuron's directional preference, implying that only visual motion directions close to the neurons' preferred direction result in c-fos expression and that near peak firing rates must be required for $c$-fos expression. In the rostral third of the $\mathrm{nBOR}$, in which 2DG studies (and unpublished electrophysiological data from our laboratory) show sensitivity essentially only to upward motion, most neurons were stained only when stimulated with upward visual motion, but not with horizontal motion stimulation, either NT or TN (compare Fig. 3A with Fig. 3C, 3E). The fact that one sees virtually no $c$-fos expression in response to motion orthogonal to the preferred motion direction implies that the level of activity present at $90^{\circ}$ to the preferred direction (approximately $45 \%$ of the maximum in the electrophysiological study of Burns (1985)) is not sufficient to induce c-fos expression.

\section{Which accessory optic neurons are stained?}

In addition to directional selectivity, LM and the $\mathrm{nBOR}$ neurons can be classified according to morphology (Bre- 
cha et al., 1980; Gamlin and Cohen, 1988), immunochemistry (Zayats et al., 2003), physiology and connectivity (Brecha et al., 1980; Pakan et al., 2006; Pakan and Wylie, 2006; Wylie et al., 2007). How these properties coalesce in functional groups is presently unclear but, presumably, neurons showing the same directional responses could differ in other aspects. For example, in both LM and nBOR, directional neurons can be further subdivided into fast and slow responsive neurons (Wylie and Crowder, 2000; Crowder et al., 2003), and evidence suggests that each subgroup projects to specific cerebellar divisions, likely playing a differential function regarding to the optokinetic response (for a thorough discussion see Pakan et al., 2006; Pakan and Wylie, 2006; Wylie et al., 2007).

To get an approximate estimate of the fraction of nBOR neurons that were stained, we counted the number of immunoreactive neurons in the nBOR of that brain that had the most stained cells for each of the four visual motion directions (diffuser switched before stimulation). The sum was $1539 c$-fos-stained neurons. Considering that we counted about a quarter of the sections in each $\mathrm{nBOR}$, this amounts to a maximum of about $50 \%$ of the 11,200 neurons in the nBOR (Peduzzi and Crossland, 1983), suggesting that many, but probably not all, accessory optic neurons can express $c$-fos under the conditions used in these experiments.

It is possible that the $c$-fos-labeled neurons were those carrying certain postsynaptic receptors. Alternatively, they may be neurons projecting to certain targets or receiving specific connections. For example, if $c$-fos expression is related to synaptic plasticity, it is plausible that this expression occurs only in those that project to the inferior olive (Brecha et al., 1980; Wylie et al., 2007) and/or those that project to the vestibular nuclei ( $37 \%$ of all cells, according to Peduzzi and Crossland, 1983), the nuclei directly driving the optokinetic nystagmus (OKN) behavior. Interestingly, Wylie (2001) and Pakan et al. (2006) have reported that LM neurons projecting to the inferior olive are located in a thin strip very much alike to the $c$-fos-stained strip of cells shown in Figs. $1 \mathrm{~F}$ and $5 \mathrm{~A}$, B. Otherwise, neurons receiving projections from the contralateral $\mathrm{nBOR}$ may be favored in conditions where the diffuser was switched, as we will argue below. Certainly, studies combining c-fos labeling with retrograde tracing and immunocytochemistry would be necessary to clarify these alternatives.

\section{c-fos induction in $\mathrm{TeO}$}

In the $\mathrm{TeO}$, we find two different $c$-fos staining patterns in the superficial, retinorecipient layers. c-fos expression was induced when the previously deprived eye was stimulated with unidirectional whole-field visual motion. The pattern of the staining was similar in response to all directions of visual motion used as stimulus, suggesting that neurons responding to different motion directions are not segregated. It is likely that this $c$-fos expression was mainly induced by the novelty of the moving stimulation to the highly motion-sensitive tectal cells. From electrophysiological studies, it is known that neurons in this region have small receptive fields and large inhibitory surrounds, with roughly $20 \%-25 \%$ of them being classical directionally selective cells (reviewed in Frost (1993).

In contrast, only when the previously deprived eye was permitted vision in the normal cage environment was $c$-fos staining seen in the deep layers of the $\mathrm{TeO}$ (layers 10 and 13). In this case the $c$-fos staining was stronger if the previously viewing eye was now covered by a diffuser. From electrophysiology, the deep layers of the $\mathrm{TeO}$ are known to be visually driven, have larger receptive fields and respond best to small moving edges [Jassik-Gerschenfeld et al., 1975; Frost and Difranco, 1976; Luksch et al., 1998, 2001, 2004]. Because some of these responses are enhanced by having their background moving in the direction opposite to the stimuli and because they respond to kinematograms, they are thought to be involved in figure-ground discrimination (Frost and Nakayama, 1983; Frost et al., 1990). It is not unreasonable that such neurons would be more strongly stimulated by the motion of other chicks in the cage than they would be by whole-field motion. The fact that the responses are weaker when the other eye is left uncovered might be attributable to fewer attentional resources being devoted to the previously deprived eye if both eyes are open. In addition, the previously deprived eye would be at disadvantage because it was probably myopic as a consequence of the deprivation (see Wallman and Winawer, 2004).

\section{Stimulus duration}

We found that approximately $1-2 \mathrm{~h}$ of continuous motion stimulus was necessary for good c-fos staining in the optokinetic nuclei. This requirement did not represent simply the time necessary for protein levels to build up to detectable levels because $30 \mathrm{~min}$ of visual stimulation followed by $90 \mathrm{~min}$ in darkness did not result in substantial $c$-fos staining, and in these cases there were even fewer stained nuclei ( $12 \%$ as many) than in birds that were exposed to visual motion stimulation for $30 \mathrm{~min}$ and then immediately sacrificed. Even bouts of $5 \mathrm{~s}$ of continuous visual motion stimulation repeated 240 times in $2 \mathrm{~h}$ did not yield $c$-fos expression. This would suggest that in order to get substantial c-fos expression, long, continuous periods of visual motion stimulation are needed (see below).

In most visual system $c$-fos studies, stimulation times are usually $1 \mathrm{~h}$. One example in which much shorter stimulation time has been demonstrated to be sufficient to induce considerable amounts of $c$-fos staining in the visual system is the study reported by Amir and Robinson (1996), in which $30 \mathrm{~s}$ of eye exposure to UV light was enough to induce $c$-fos expression in the visual cortex of the rat. In other systems, however, shorter stimulation times do result in substantial $c$-fos expression, provided that enough time is allowed for the protein to build up. For example, 10 pulses of radiant heat, each of $20 \mathrm{~s}$ duration, given at $90 \mathrm{~s}$ intervals to the rat hind paw results in substantial $c$-fos immunoreactivity in spinal dorsal horn neurons (Hunt et al., 1987). Also, in the song-learning system of songbirds, as little as $2 \mathrm{~s}$ of exposure to sound can produce robust immediate-early gene expression (Kruse et al., 2000). 
Novelty requirement for $c$-fos expression and neural plasticity

Our results support the assertion that $c$-fos expression in neurons of sensory systems requires stimuli not usually experienced, or "novel," in situations calling for neuronal plasticity. As mentioned above, "novelty" after visual deprivation was crucial to obtain c-fos staining in the superficial and deep layers of the TeO. In the nBOR, we found the highest number of $c$-fos expressing cells after exposing the previously deprived eye to continuous whole-field visual motion. In this situation the "novelty" produced by the abnormal continuous motion is enhanced by the previous history of visual deprivation. In the LM, continuous visual stimulation was enough to obtain a strong c-fos expression. We suppose that the optokinetic nuclei, like the vestibular nuclei, regard signals of continuous rotation as though the neural signals defining zero rotation are miscalibrated and need adjustment. Contrariwise, brief periods of whole-field unidirectional optic flow occur whenever an animal turns or moves through its environment and thus are the normal experience of neurons in the visual system. Such stimuli do not signify a need to adjust synaptic strength and therefore are not a sufficient perturbation to provoke $c$-fos expression. In this way the stimulus situation we have imposed resembles that experienced after a unilateral vestibular lesion, in which the unidirectional nystagmus gradually abates as the zero-motion condition becomes redefined. It has been shown that continuous whole-field rotations like the ones used in this study increase or decrease the gain of the optokinetic response depending on stimulus duration (see Collewijn, 1985).

Additional support for the notion that long periods of rotational visual stimulation can cause changes in the response to different directions of input comes from work on changes in the asymmetry of OKN after such stimulation. Normally, if the left eye is covered, horizontal rotational visual stimulation to the left causes much stronger OKN than to the right. Thus during a head turn, the right eye drives the OKN during rightward turns and the left eye during leftward turns. If, however, one eye is covered for a long period, the asymmetry between the two directions is reduced, so that the open eye can mediate OKN in both directions (Yücel et al., 1990).

We find it intriguing, however, that continuous visual motion induces little $c$-fos expression in the nBOR contralateral to the open eye if the diffuser is left on the covered eye, compared to the situations in which the diffuser on the other eye is removed before the visual stimulation. In both cases the stimulated eye received the same continuous visual motion stimulation as did the open eye in the birds wearing a diffuser, and the main difference seems to be in the experience of the fellow eye. Why then should the degree of $c$-fos staining differ? We conjecture that the indirect inputs from the contralateral eye are important to compute the abnormal or novel condition produced by the continuous whole-field rotation. Support for this conjecture comes from the finding that there are neurons in the $\mathrm{nBOR}$ that receives input from the contralateral
nBOR (Brecha et al., 1980; Wylie et al., 1997, 2007), and many $\mathrm{nBOR}$ neurons show binocular responses which, by combining appropriate directions of visual stimulation in the two eyes, explicitly encode rotations and translations (Wylie et al., 1990b, 1999). The fact that monocular stimulation of nondeprived chicks produced strong c-fos expression, also indicates that changes in the signals coming from the unstimulated eye are relevant to define a novel situation.

Our findings are broadly consistent with results from several other studies that suggest that $c$-fos expression is related to stimuli being novel and/or conducive to plastic changes (Anokhin and Rose, 1991; Kaczmarek and Chaudhuri, 1997; Svarnik et al., 2005). Basal levels of c-fos expression are generally very low, even in areas with high levels of spontaneous neuronal activity, like the visual cortex (Kaczmarek and Chaudhuri, 1997; Mower, 1994), but exposure to novel sensory stimuli rapidly results in a transient increase both in mRNA and protein levels in a variety of systems (Staiger et al., 2002; Carretta et al., 1999; Beaver et al., 1993; Anokhin et al., 1991; Hunt et al., 1987; Mower and Kaplan, 1999, 2002). Additional evidence for a role of $c$-fos and other immediate-early genes in plasticity comes from behavioral studies that have found enhanced c-fos expression in animals that are learning a task (Anokhin et al., 1991; Dragunow, 1996; Tischmeyer and Grimm, 1999; Schettino and Otto, 2001; Guzowski et al., 2001; Svarnik et al., 2005).

\section{CONCLUSION}

In conclusion, $c$-fos expression in the chick visual system is induced by stimuli that optimally excite the AOS and pretectal neurons. A high degree of stimulation, however, is not sufficient; the stimulus situation must be novel and of sufficiently long duration. Our results support the assumption that this expression is principally related to stimulus circumstances leading to long-term changes in neuronal functioning.

Acknowledgments-We thank Dr. John Leah for invaluable help in the beginning of this study, and Dr. Rodrigo Bravo for the c-fos antibody. This work was supported by NSF grant BNS 9108459 and NIH RR03060. Gonzalo Marin was supported by FONDECYT grant 1080220.

\section{REFERENCES}

Amir S, Robinson B (1996) Fos expression in rat visual cortex induced by ocular input of ultraviolet light. Brain Res 716:213-218.

Anokhin KV, Mileusnic R, Shamakhina I, Rose SPR (1991) Effects of early experience on c-fos gene expression in the chick forebrain. Brain Res 544:101-107.

Anokhin KV, Rose SPR (1991) Learning-induced increase of immediate early gene messenger RNA in the chick forebrain. Eur $\mathrm{J}$ Neurosci 3:162-167.

Bisler S, Schleicher A, Gass P, Stehle JH, Zilles K, Staiger JF (2002) Expression of c-Fos, ICER, Krox-24 and JunB in the whisker-tobarrel pathway of rats: time course of induction upon whisker stimulation by tactile exploration of an enriched environment. J Chem Neuroanat 23:187-198. 
Beaver CJ, Mitchell DE, Robertson HA (1993) Immunohistochemical study of the pattern of rapid expression of $\mathrm{c}$-fos protein in the visual cortex of dark-reared kittens following initial exposure to light. J Comp Neurol 333:469-484.

Bodnarenko SR, Rojas X, McKenna OC (1988) Spatial organization of the retinal projection to the avian lentiform nucleus of the mesencephalon. J Comp Neurol 269:431-447.

Brecha N, Karten HJ, Hunt SP (1980) Projections of the nucleus of the basal optic root in the pigeon: an autoradiographic and horseradish peroxidase study. J Comp Neurol 189:615-670.

Burns S (1985) The avian accessory optic system: neurophysiology, development and oculomotor function. PhD dissertation, City University of New York, New York, NY.

Burns S, Wallman J (1981) Relation of single unit properties to the oculomotor function of the nucleus of the basal optic root (accessory optic system) in chickens. Exp Brain Res 42:171-180.

Carretta D, Hervé-Minvelle A, Bajo VM, Villa AEP, Rouiller EM (1999) Preferential induction of fos-like immunoreactivity in granule cells of the cochlear nucleus by acoustic stimulation in behaving rats. Neurosci Lett 259:123-126.

Cole AJ, Saffen DW, Baraban JM, Worley PF (1989) Rapid increase of an immediate early gene messenger RNA in hippocampal neurons by synaptic NMDA receptor activation. Nature 340:474-476.

Collewijn H (1985) Integration of adaptive changes of the optokinetic reflex, pursuit and the vestibulo-ocular reflex. Rev Oculomot Res $1: 51-69$.

Crowder NA, Dawson M, Wylie DR (2003) Temporal frequency and velocity-like tuning in the pigeon accessory optic system. J Neurophysiol 90:1829-1841.

Curran T, Franza BR (1988) Fos and Jun: the AP-1 connection. Cell 55:395-397.

Dragunow M (1996) A role for immediate-early transcription factors in learning and memory. Behav Genet 26:293-299.

Dragunow M, Faull R (1989) The use of c-fos as a metabolic marker in neuronal pathway tracing. J Neurosci Methods 29:261-265.

Ehrlich D, Mark R (1984) An atlas of the primary visual projections in the brain of the chick, Gallus gallus. J Comp Neurol 223:592-610.

Frost BJ (1993) Subcortical analysis of visual motion: relative motion, figure-ground discrimination and self-induced optic flow. Rev Oculomot Res 5:159-175.

Frost BJ, di Franco DE (1976) Motion characteristics of single units in the pigeon optic tectum. Vis Res 16:1229-1234.

Frost BJ, Nakayama K (1983) Single visual neurons code opposing motion independent of direction. Science 220:744-745.

Frost BJ, Wylie DR, Wang WC (1990) The processing of object and self-motion in the tectofugal and accessory optic pathways of birds. Vis Res 30(11):1677-1688.

Fu YX, Gao HF, Guo MW, Wang SR (1998) Receptive field properties of visual neurons in the avian nucleus lentiformis mesencephali. Exp Brain Res 118:279-285.

Gamlin PD, Cohen DH (1988) Retinal projections to the pretectum in the pigeon (Columba livia). J Comp Neurol 269:1-17.

Gilman MZ, Berkowitz LA, Feramisco JR, Franza BR Jr, Graham RM, Riabowol KT, Ryan WA Jr (1988) Intracellular mediators of c-fos induction. Cold Spring Harb Symp Quant Biol (Pt 2) 53:761-767.

Guzowski JF, Setlow B, Wagner EK, McGaugh JL (2001) Experiencedependent gene expression in the rat hippocampus after spatial learning: a comparison of the immediate-early genes arc, c-fos, and zif268. J Neurosci 21:5089-5098.

Herdegen T, Leah JD (1998) Inducible and constitutive transcription factors in the mammalian nervous system: control of gene expression by Jun, Fos and Krox, and CREB/ATF proteins. Brain Res Rev 28:370-490.

Hossokawa NM, Araki CM, Hamassaki-Britto DE, Wallman J, Britto LR (1996) Expression of the Fos protein reveals functional subdivisions of the avian ventral lateral geniculate nucleus. Neurosci Lett 218:53-56.
Hunt SP, Pini A, Evan G (1987) Induction of c-fos-like protein in spinal cord neurons following sensory stimulation. Nature 328:632-634.

Illig KR, Haberly LB (2003) Odor-evoked activity is spatially distributed in piriform cortex. J Comp Neurol 457:361-373.

Illig KR (2007) Developmental changes in odor-evoked activity in rat piriform cortex. Neuroscience 145:370-376.

Jassik-Gerschenfeld D, Guichard J, Tessier Y (1975) Localization of directionally selective and movement sensitive cells in the optic tectum of the pigeon. Vis Res 15:1037-1038.

Kaczmarek L, Chaudhuri A (1997) Sensory regulation of immediate early gene expression in mammalian visual cortex: implications for functional mapping and neural plasticity. Brain Res Rev 23:237256.

Kovary K, Bravo R (1991) The jun and fos protein families are both required for cell cycle progression in fibroblasts. Mol Cell Biol 11: $4466-4472$.

Kruse AA, Stripling R, Clayton DF (2000) Minimal experience required for immediate-early gene induction in zebra finch neostriatum. Neurobiol Learn Mem 74:179-184.

Kuhlenbeck $H$ (1939) The development and structure of the pretectal cell masses in the chick. J Comp Neurol 71:361-387.

Leah JD, Herdegen T, Bravo R (1991) Selective expression of Jun proteins following axotomy and axonal transport block in peripheral nerves in the rat: evidence for a role in the regeneration process. Brain Res 566:198-207.

Luksch H, Cox K, Karten HJ (1998) Bottlebrush dendritic endings and large dendritic fields: motion-detecting neurons in the tectofugal pathway. J Comp Neurol 396:399-414.

Luksch H, Karten HJ, Kleinfeld D, Wessel R (2001) Chattering and differential signal processing in identified motion-sensitive neurons of parallel visual pathways in the chick tectum. J Neurosci 21: $6440-6446$

Luksch H, Khanbabaie R, Wessel R (2004) Synaptic dynamics mediate sensitivity to motion independent of stimulus details. Nat Neurosci 7:380-388.

McKenna OC, Wallman J (1981) Identification of avian brain regions responsive to retinal slip using 2-deoxyglucose. Brain Res 210: 455-460.

McKenna OC, Wallman J (1985a) Accessory optic system and pretectum of birds: comparisons with those of other vertebrates. Brain Behav Evol 26:91-116.

McKenna OC, Wallman J (1985b) Functional postnatal changes in avian brain regions responsive to retinal slip: a 2-deoxy-D-glucose study. J Neurosci 5:330-342.

Melzer P, Steiner H (1997) Stimulus-dependent expression of immediate-early genes in rat somatosensory cortex. J Comp Neurol 380:145-153.

Montero VM, Jian S (1995) Induction of c-fos protein by patterned visual stimulation in central visual pathways of the rat. Brain Res 690:189-199.

Morgan JI, Curran T (1986) Role of ion flux in the control of c-fos expression. Nature 322:552-555.

Morgan JI, Curran T (1991) Stimulus-transcription coupling in the nervous system: involvement of the inducible proto-oncogenes fos and jun. Annu Rev Neurosci 14:421-451.

Morgan B, Frost BJ (1981) Visual responses characteristics of neurons in nucleus of basal optic root of pigeons. Exp Brain Res 42:181-188.

Mower GD (1994) Differences in the induction of Fos protein in cat visual cortex during and after the critical period. Mol Brain Res 21:47-54.

Mower GD, Kaplan IV (1999) Fos expression during the critical period in visual cortex: differences between normal and dark reared cats. Mol Brain Res 64:264-269.

Mower GD, Kaplan IV (2002) Immediate early gene expression in the visual cortex of normal and dark reared cats: differences between fos and egr-1. Mol Brain Res 105:157-160. 
Natal CL, Letelier JC, Rojas X, Wallman J (1989) Visual deprivation changes directional preference of accessory optic system (AOS) neurons in chickens. Soc Neurosci Abstr 15:460.

Pakan JM, Wylie DR (2006) Two optic flow pathways from the pretectal nucleus lentiformis mesencephali to the cerebellum in pigeons (Columba livia). J Comp Neurol 499:732-744.

Pakan JM, Krueger K, Kelcher E, Cooper S, Todd KG, Wylie DR (2006) Projections of the nucleus lentiformis mesencephali in pigeons (Columba livia): a comparison of the morphology and distribution of neurons with different efferent projections. J Comp Neurol 495:84-99.

Peduzzi JD, Crossland WJ (1983) Morphology of normal and deafferented neurons in the chick ectomamillary nucleus. J Comp Neurol 213:301-309.

Sagar SM, Sharp FR, Curran T (1988) Expression of the c-fos protein in brain: metabolic mapping at the cellular level. Science 240: $1328-1331$.

Schettino LF, Otto T (2001) Patterns of Fos expression in the amygdala and ventral perirhinal cortex induced by training in an olfactory fear conditioning paradigm. Behav Neurosci 115:1257-1272.

Sheng M, McFadden G, Greenberg ME (1990) Membrane depolarization and calcium induce c-fos transcription via phosphorylation of transcription factor CREB. Neuron 4:571-582.

Staiger J-F, Masanneck CS, Bisler S, Schleicher A, Zuschratter W, Zilles K (2002) Excitatory and inhibitory neurons express c-fos in barrel-related columns after exploration of a novel environment. Neuroscience 109:687-699.

Svarnik OE, Alexandrov YI, Gavrilov VV, Grinchenko YV, Anokhin KV (2005) Fos expression and task-related neuronal activity in rat cerebral cortex after instrumental learning. Neuroscience 136: 33-42.

Thompson MA, Ginty DD, Bonni A, Greenberg ME (1995) L-type voltage-sensitive $\mathrm{Ca}^{2+}$ channel activation regulates c-fos transcription at multiple levels. J Biol Chem 270:4224-4235.

Telford L, Frost BJ (1989) Functional activity in the accessory optic system during visual, vestibular and visual-vestibular stimulation in the pigeon. Exp Brain Res 77:391-397.

Tischmeyer W, Grimm R (1999) Activation of immediate early genes and memory formation. Cell Mol Life Sci 55:564-574.

Wallman J (1993) Subcortical optokinetic mechanisms. Rev Oculomot Res 5:321-342.

Wallman J, Turkel J, Trachtman J (1978) Extreme myopia produced by modest change in early visual experience. Science 201:12491251.

Wallman J, Winawer J (2004) Homeostasis of eye growth and the question of myopia. Neuron 43:447-468.
Wallman J, Saldanha CJ, Silver R (1994) A putative suprachiasmatic nucleus of birds responds to visual-motion. J Comp Physiol A 174:297-304.

Winterson BJ, Brauth SE (1985) Direction selective single units in the nucleus lentiformis mesencephali of the pigeon (Columba livia). Exp Brain Res 60:215-226.

Wylie DR, Crowder NA (2000) Spatiotemporal properties of fast and slow neurons in the pretectal nucleus lentiformis mesencephali in pigeons. J Neurophysiol 84:2529-2540.

Wylie DR, Frost BJ (1990a) Visual response properties of neurons in the nucleus of the basal optic root of the pigeon: a quantitative analysis. Exp Brain Res 82:327-336.

Wylie DR, Frost BJ (1990b) Binocular neurons in the nucleus of the basal optici root (nBOR) of the pigeon are selective for either translational or rotational visual flow. Vis Neurosci 5:489-495.

Wylie DRW, Frost BJ (1996) The pigeon optokinetic system—visual input in extraocular-muscle coordinates. Vis Neurosci 13:945-953.

Wylie DR, Linkenhoker B, Lau KL (1997) Projections of the nucleus of the basal optic root in pigeons (Columba livia) revealed with biotinylated dextran amine. J Comp Neurol 384:517-536.

Wylie DR, Frost BJ (1999) Responses of neurons in the nucleus of the basal optic root to translational and rotational flowfields. J Neurophysiol 81:267-276.

Wylie DR (2001) Projections from the nucleus of the basal optic root and nucleus lentiformis mesencephali to the inferior olive in pigeons (Columba livia). J Comp Neurol 429:502-513.

Wylie DR, Pakan JM, Elliott CA, Graham DJ, Iwaniuk AN (2007) Projections of the nucleus of the basal optic root in pigeons (Columba livia): a comparison of the morphology and distribution of neurons with different efferent projections. Vis Neurosci 24:691707.

Yücel YH, Kim MS, Jardon B, Bonaventure N (1990) Abolition of monocular optokinetic nystagmus directional asymmetry after unilateral visual deprivation in adult vertebrates: involvement of the GABAergic mechanism. Brain Res Dev Brain Res 53:179-185.

Zayats N, Eyre MD, Nemeh A, Tombol T (2003) The intrinsic organization of the nucleus lentiformis mesencephali magnocellularis: a light- and electron-microscopic examination. Cells Tissues Organs 174:194-207.

Zhang T, Fu YX, Hu J, Wang SR (1999) Receptive field characteristics of neurons in the nucleus of the basal optic root in pigeons. Neuroscience 91:33-40.

Zhao R, Liu L, Rittenhouse AR (2007) $\mathrm{Ca}^{2+}$ influx through both L- and $\mathrm{N}$-type $\mathrm{Ca}^{2+}$ channels increases c-fos expression by electrical stimulation of sympathetic neurons. Eur J Neurosci 25:1127-1135.

Zou Z, Li F, Buck LB (2005) Odor maps in the olfactory cortex. Proc Natl Acad Sci U S A 102:7724-7729. 